\title{
THE HOMOLOGY OF THE CLASSICAL GROUPS OVER THE DYER-LASHOF ALGEBRA ${ }^{1,2}$
}

\author{
BY STANLEY O. KOCHMAN
}

Communicated by N. E. Steenrod, July 1, 1970

1. Introduction. By Bott periodicity the classical groups, their classifying spaces, their homogeneous spaces, $\operatorname{Im} J$ and $B \operatorname{Im} J$ are infinite loop spaces, and hence the Dyer-Lashof operations act on their $\bmod p$ homology for $p$ a prime number. We will list the basic properties of the Dyer-Lashof operations in $\S 2$. These operations have been calculated in the homology of all these spaces, and the dual operations have also been computed. As applications one can calculate the indecomposable elements of the homology of these spaces over the Dyer-Lashof algebra R and the AR-Hopf algebra maps between any two classical groups or any two of their classifying spaces. In this paper we will summarize our results for $B U, \mathrm{BU}$ (the connected and infinite component versions of the classifying space of the infinite unitary group), $O, S O, B \operatorname{Im} J$ and $\operatorname{Im} J$.

These results have been applied to the study of $H_{*}(F)$ and $H_{*}(B F)$ by J. P. May [9] and I. Madsen [7]. They are also useful in cobordism theory (see for example T. tom Dieck [5, p. 396]).

The author is very grateful to J. Peter May for his guidance, suggestions and generosity with his time throughout the preparation of this paper.

Notation. All elements of a graded object will be indexed by their degree with the exception of the Chern and Wu classes. All homology and cohomology will have $Z_{p}$ coefficients for $p$ a prime number. When a result differs for $p$ an odd prime and $p=2$ then the result for $p=2$ will be placed in square brackets.

2. The Dyer-Lashof operations. The homology of an infinite loop space $B$ has natural homomorphisms $Q^{i}: H_{*}(B) \rightarrow H_{*}(B)$ for $i \geqq 0$ of degree $2 i(p-1)$ [of degree $i$ ] which have been studied by S. Araki and

AMS 1970 subject classifications. Primary 55F45; Secondary 55G99.

Key words and phrases. Classical groups, classifying space, infinite loop space, Dyer-Lashof algebra, Bott periodicity, suspension, Nishida relations, image of J.

1 During the preparation of this paper the author was supported by a National Science Foundation Graduate Fellowship.

2 The work announced here is contained in the author's doctoral thesis, submitted to the University of Chicago. 
T. Kudo [3], W. Browder [4], E. Dyer and R. Lashof [6], G. Nishida [10] and J. P. May [8]. Theorem 1 summarizes some of their results.

THEOREM 1. The Dyer-Lashof operations satisfy the following propperties:

(a) $Q^{0}(\phi)=\phi$ and $Q^{i}(\phi)=0$ if $i>0$ where $\phi \in H_{0}(B)$ is the identity element for the loop product in $H_{*}(B)$.

(b) $Q^{i}(x)=0$ if $2 i<\operatorname{deg} x$ [if $\left.i<\operatorname{deg} x\right]$.

(c) $Q^{i}(x)=x^{p}$ if $2 i=\operatorname{deg} x[$ if $i=\operatorname{deg} x]$.

(d) $\sigma_{*} \circ Q^{i}=Q^{i} \circ \sigma_{*}$ where $\sigma_{*}: I H_{*}(\Omega B) \rightarrow H_{*}(B)$ is the homology suspension map.

(e) (Multiplicative Cartan formula) $Q^{r}(x y)=\sum_{i=0}^{r} Q^{i}(x) Q^{r-i}(y)$.

(f) (Comultiplicative Cartan formula)

$$
\psi \circ Q^{r}(x)=\sum_{i=0}^{r} \sum Q^{i}\left(x^{\prime}\right) \otimes Q^{r-i}\left(x^{\prime \prime}\right)
$$

where $\psi(x)=\sum x^{\prime} \otimes x^{\prime \prime}$.

(g) $\chi \circ Q^{i}=Q^{i} \bigcirc \chi$ where $\chi$ is the conjugation of $H_{*}(B)$.

(h) (Nishida relations) Let $P_{*}^{s}: H_{*}(B) \rightarrow H_{*}(B)$ of degree $-2 s(p-1)$ [of degree $-s$ ] be dual to the Steenrod operation $P^{s}$. Then

$$
P_{*}^{s} \circ Q^{r}=\sum(-1)^{i+s}(s-p i, r(p-1)-p s+p i) Q^{r-s+i} \circ P_{*}^{i} .
$$

We use the convention $(i, j)=(i+j) ! / i ! j !$ if $i \geqq 0$ and $j \geqq 0$ while $(i, j)=0$ if $i<0$ or $j<0$. Thus the above sum is taken over all integers $i$.

(i) (Adem relations) If $a>p b$ then

$$
Q^{a} \circ Q^{b}=\sum(-1)^{a+i}(p i-a, a-(p-1) b-i-1) Q^{a+b-i} \circ Q^{i} .
$$

Definition 2. The Dyer-Lashof algebra $R$ is the quotient algebra $F / J$ where $F$ is the free associative algebra generated by $\left\{Q^{r}, \beta Q^{r+1} \mid r \geqq 0\right\}$ [by $\left\{Q^{r} \mid r \geqq 0\right\}$ ], and $J$ is the ideal of $F$ consisting of all elements which annihilate every element of every infinite loop space.

3. Results on $B U$ and BU. Recall that $H^{*}(B U)=P\left\{c_{n} \mid n \geqq 1\right\}$ as algebras where $c_{n}$ is the Chern class of degree $2 n, c_{0}=1$ and $\psi\left(c_{n}\right)$ $=\sum_{i=0}^{n} c_{i} \otimes c_{n-i}$. Let $a_{2 n}=\left(c_{1}^{n}\right)^{*}$ and $\mathrm{p}_{2 n}=c_{n}^{*}$ in the dual basis of the basis of $H^{*}(B U)$ which consists of monomials in the Chern classes. Then $H_{*}(B U)=P\left\{a_{2 n} \mid n \geqq 1\right\}$ as algebras with $\psi\left(a_{2 n}\right)$ $=\sum_{i=0}^{n} a_{2 i} \otimes a_{2 n-2 i}$, and $\left\{\mathrm{p}_{2 n} \mid n \geqq 1\right\}$ is a basis for the primitive elements of $H_{*}(B U)$. For $r \geqq 0$ we have the duals of the Dyer-Lashof operations $Q_{*}^{r}: H^{*}(B U) \rightarrow H^{*}(B U)$ of degree $-2 r(p-1)$ [of degree 
$-r$. Theorems 3 and 4 are our main results on $B U$.

THEOREM 3. In $H_{*}(B U)$ for $r \geqq 0$ and $n \geqq 1$,

$$
\begin{aligned}
Q^{r}\left(\mathrm{p}_{2 n}\right) & =(-1)^{r+n}(n-1, r-n) \mathrm{p}_{2 n+2 r(p-1)} \\
{\left[Q^{2 r}\left(\mathrm{p}_{2 n}\right)\right.} & \left.=(n-1, r-n) \mathrm{p}_{2 n+2 r}\right],
\end{aligned}
$$

and

$$
\begin{aligned}
Q^{r}\left(a_{2 n}\right) & =(-1)^{r+n+1}(n, r-n-1) a_{2 n+2 r(p-1)}+\text { decomposables } \\
{\left[Q^{2 r}\left(a_{2 n}\right)\right.} & \left.=(n, r-n-1) a_{2 n+2 r}+\text { decomposables }\right] .
\end{aligned}
$$

THEOREM 4. In $H^{*}(B U)$ for $r \geqq 0$ and $n \geqq 1$,

$$
\begin{aligned}
Q_{*}^{r}\left(c_{n}\right) & =(-1)^{r+n}(n-r(p-1)-1, p r-n) c_{n-r(p-1)} \\
{\left[Q_{*}^{2 r}\left(c_{n}\right)\right.} & \left.=(n-r-1,2 r-n) c_{n-r}\right] .
\end{aligned}
$$

Theorem 3 is proved by using the comultiplicative Cartan formula, the Nishida relations and Bott periodicity. An algorithm can then be given for computing $Q^{r}\left(a_{2 n}\right)\left[Q^{2 r}\left(a_{2 n}\right)\right]$ by induction on $n+r(p-1)$ and for fixed $n+r(p-1)$ by induction on $n$ using the following seven properties that the $Q^{r}$ satisfy on $H_{*}(B U):(\mathrm{a}),(\mathrm{b}),(\mathrm{c}),(\mathrm{e}),(\mathrm{f}),(\mathrm{h})$ of Theorem 1 and Theorem 3 . Theorem 4 is now proved by defining homomorphisms $R_{*}^{s}$ on $H^{*}(B U)$ by

$$
\begin{aligned}
R_{*}^{s}\left(c_{n}\right) & =(-1)^{s+n}(n-s(p-1)-1, p s-n) c_{n-s(p-1)} \\
{\left[R_{*}^{2 s}\left(c_{n}\right)\right.} & \left.=(n-s-1,2 s-n) c_{n-s}\right]
\end{aligned}
$$

and insisting that the $R_{*}^{s}$ satisfy the multiplicative Cartan formula. Then the dual maps $R^{8}$ can be shown to satisfy the above seven properties. Hence $R^{s}=Q^{s}$ for all $s \geqq 0$ by the algorithm for computing the Dyer-Lashof operations on $H_{*}(B U)$.

Recall that $H_{*}(\mathrm{BU})=H_{*}(B U) \otimes Z_{p}(Z)$ as Hopf algebras where $Z_{p}(Z)$ is the group algebra of the integers over $Z_{p}$. Elements of $H_{*}(\mathrm{BU})$ are written $x \otimes[i]$ for $x \in H_{*}(B U)$ and $i \in Z \subset Z_{p}(Z)$. Since the canonical inclusion $B U \rightarrow B U$ induces the map $x \rightarrow x \otimes[0]$ in homology for $x \in H_{*}(B U)$, the following theorem together with our knowledge of the Dyer-Lashof operations on $H_{*}(B U)$ tell us how the Dyer-Lashof operations act on $H_{*}(\mathrm{BU})$.

THEOREM 5. In $H_{*}(\mathrm{BU})$ for $n \geqq 1, Q^{n}(1 \otimes[1])=\gamma_{n}\left(\mathrm{p}_{2(p-1)}\right) \otimes[p]$ $\left[Q^{2 n}(1 \otimes[1])=a_{2 n} \otimes[2]\right]$ where $\gamma_{n}\left(\mathrm{p}_{2(p-1)}\right)$ is $\left(c_{p-1}^{n}\right)^{*}$ in the dual basis of the basis of $H^{*}(B U)$ which consists of monomials in the Chern classes. 
We will now sketch the applications of the preceding theorems. Define the R-indecomposables of $H_{*}(B U)$ by

$$
Q_{\mathrm{R}} H_{*}(B U)=I H_{*}(B U) /\left[(I \mathrm{R})\left(I H_{*}(B U)\right)+\left(I H_{*}(B U)\right)^{2}\right] .
$$

The following theorem is a consequence of Theorem 3.

THEOREM 6. $\left\{a_{2 k} \mid k \geqq 1, k=p^{e} h, h=s(p-1)+r, 1 \leqq r \leqq p-1, s\right.$ $=\sum_{i=0}^{n} s_{i} p^{i}, 0 \leqq s_{i} \leqq p-1, s_{i}=s_{i}$ if $s_{i} \neq 0, s_{i}=p$ if $s_{i}=0$ and if $s \neq 0$ then $s_{n} \neq 0$ and $\left.r \geqq s_{0} \geqq \cdots \geqq \bar{s}_{n}\right\} \quad\left[\left\{a_{2^{k}} \mid k \geqq 0\right\}\right]$ is a $Z_{p}$-basis for $Q_{\mathrm{R}} H_{*}(B U)$.

If $A$ is a connected Hopf algebra and $f: A \rightarrow A$ is a morphism of Hopf algebras then $f$ is said to be locally nilpotent if for all $a \in A$ with $\operatorname{deg} a>0$ there is a positive integer $n(a)$ with $f^{n(a)}(a)=0$ where $f^{0}=1$, $f^{1}=f$ and $f^{k+1}=f \circ f^{k}$ for $k \geqq 1$. In this case every element of $Z_{p}[[f]]$ is a well-defined Hopf algebra endomorphism of $A$ where addition is given by Whitney sum, i.e., $g+h=\Phi \circ g \otimes h \circ \Psi$. The composite map $\phi: B U \rightarrow B S p \rightarrow B U$ induces a locally nilpotent endomorphism $\phi_{*}$ of $H_{*}\left(B U ; Z_{2}\right) . Z_{2}\left[\left[\phi_{*}\right]\right]$ is the ring of all A-Hopf algebra endomorphisms of $H_{*}\left(B U ; Z_{2}\right)$ and the ring of all AR-Hopf algebra endomorphisms of $H_{*}\left(B U ; Z_{2}\right)$. If $p$ is odd then $B U_{p}=\prod_{i=0}^{p-2} B U_{p, i}$ as infinite loop spaces by J. F. Adams [1, Lecture 4]. There is a locally nilpotent AR-Hopf algebra endomorphism $\phi_{i}$ of $H_{*}\left(B U_{p, i}\right)$ for $0 \leqq i \leqq p-2$ such that $Z_{p}\left[\left[\phi_{i}\right]\right]$ is the ring of all A-Hopf algebra endomorphisms of $H_{*}\left(B U_{p, i}\right)$ and the ring of all AR-Hopf algebra endomorphisms of $H_{*}\left(B U_{p, i}\right)$.

4. Results on $O$ and $S O$. Recall that $H_{*}\left(S O ; Z_{p}\right)=E\left\{d_{4 n+8} \mid n \geqq 0\right\}$ as Hopf algebras if $p$ is an odd prime and $H_{*}\left(S O ; Z_{2}\right)=E\left\{u_{n} \mid n \geqq 1\right\}$ as algebras with $u_{0}=1$ and $\psi\left(u_{n}\right)=\sum_{i=0}^{n} u_{i} \otimes u_{n-i}$. Hence $H^{*}\left(S O ; Z_{p}\right)$ $=E\left\{d_{4 n+3}^{*} \mid n \geqq 0\right\}$ as Hopf algebras if $p$ is an odd prime and $H^{*}\left(S O ; Z_{2}\right)$ $=P\left\{e_{2 n+1} \mid n \geqq 0\right\}$ as Hopf algebras. We use the convention that the $(n+1)$ st Pontryagin class suspends to $-d_{4 n+3}^{*}$. Let $\left\{p_{2 n-1} \mid n \geqq 1\right\}$ be the primitive elements of $H_{*}\left(S O ; Z_{2}\right)$. From the analogue of Theorem 4 for $H^{*}(B O)$ we deduce the following three results by using the cohomology suspension map.

TheOREM 7. In $H^{*}(S O)$ for $r \geqq 0$ and $n \geqq 0$,

$$
\begin{aligned}
Q_{*}^{r}\left(d_{4 n+3}^{*}\right) & =(-1)^{r}(2 n-r(p-1)+1, p r-2 n-2) d_{4 n-2 r(p-1)+3}^{*} \\
{\left[Q_{*}^{2 r}\left(e_{2 n+1}\right)\right.} & \left.=(n-r, 2 r-n-1) e_{2 n-2 r+1}\right] .
\end{aligned}
$$

Corollary 8. In $H_{*}\left(S O ; Z_{p}\right)$ for $p$ an odd prime, $r \geqq 0$ and $n \geqq 0$, 


$$
Q^{r}\left(d_{4 n+3}\right)=(-1)^{r}(2 n+1, r-2 n-2) d_{4 n+2 r(p-1)+3} .
$$

CoROllary 9. In $H_{*}\left(S O ; Z_{2}\right)$ for $r \geqq 0$ and $n \geqq 1$,

$$
Q^{r}\left(u_{n}\right)=(n, r-n-1) u_{r+n}+\text { decomposables }
$$

and

$$
Q^{2 r}\left(\mathrm{p}_{2 n-1}\right)=(n-1, r-n) \mathrm{p}_{2 n+2 r-1} .
$$

ThEOREM 10. In $H_{*}\left(S O ; Z_{2}\right)$ for $r \geqq 0$ and $n \geqq 1$,

$$
Q^{r}\left(u_{n}\right)=\sum_{a+b+c=r+n}(n-a, r-n-b-1) u_{a} u_{b} u_{c} .
$$

Proof. This theorem follows from the first assertion of Corollary 9, the comultiplicative Cartan formula and the binomial coefficient identities of J. Adem [2, p. 233].

Recall that $H_{*}\left(O ; Z_{2}\right)=H_{*}\left(S O ; Z_{2}\right) \otimes Z_{2}\left(Z_{2}\right)$ as Hopf algebras. For $x \in H_{*}\left(S O ; Z_{2}\right)$ write $x$ and $x \otimes[-1]$ to designate elements in the two components of $H_{*}(0)$. The multiplicative Cartan formula, Theorem 10 and the following theorem give complete information on the DyerLashof operations on $H_{*}\left(O ; Z_{2}\right)$.

TheOREM 11. In $H_{*}\left(O ; Z_{2}\right), Q^{n}(1 \otimes[-1])=u_{n}$ for $n \geqq 0$.

5. Results on $B \operatorname{Im} J$ and $\operatorname{Im} J$. Throughout this section $p$ will be an odd prime. J. Stasheff [11] has constructed infinite loop spaces $B \operatorname{Im} J$ and $\operatorname{Im} J$. He has shown that $H^{*}(B \operatorname{Im} J)=H^{*}(B O)_{p, 0}$ $\otimes H^{*}(S U)_{p, 0}$ and $H^{*}(\operatorname{Im} J)=H^{*}(B O)_{p, 0} \otimes H^{*}(S O)_{p, 1 / 2(p-3)}$ as Hopf algebras with each factor closed under $p^{r}$, for all $r \geqq 0$. Recall that $H^{*}(B O)_{p, 0}=P\left\{q_{n} \mid n \geqq 1\right\}$ as algebras with deg $q_{n}=2 n(p-1), q_{0}=1$ and $\psi\left(q_{n}\right)=\sum_{i=0}^{n} q_{i} \otimes q_{n-i}$. Then $q_{n}$ are the Wu classes which are defined by $q_{n}=\Phi^{-1} \circ P^{n} \circ \Phi(1)$ where $\Phi$ is the Thom isomorphism.

TheOREM 12. Let $p$ be an odd prime. Then

$$
H^{*}(B \operatorname{Im} J)=H^{*}(B O)_{p, 0} \otimes H^{*}(S U)_{p, 0}
$$

and

$$
H^{*}(\operatorname{Im} J)=H^{*}(B O)_{p, 0} \otimes H^{*}(S O)_{p, 1 / 2(p-3)}
$$

as Hopf algebras with each factor closed under $P^{r}$ and $Q^{r}$, for all $r \geqq 0$. In particular for $r \geqq 0$ and $n \geqq 1$,

$$
Q_{*}^{r}\left(q_{n}\right)=((n-r)(p-1)-1, p r-n(p-1)) q_{n-r} .
$$

Detailed proofs and statements of all the above results and many similar ones will appear elsewhere. 


\section{BIBLIOGRAPHY}

1. J. F. Adams, Lectures on generalised cohomology, Lecture Notes in Math., no. 99, Springer-Verlag, New York, 1969.

2. J. Adem, The relations on Steenrod powers of cohomology classes, Algebraic Geometry and Topology (A Symposium in Honor of S. Lefschetz) Princeton Univ. Press, Princeton, N. J., 1957, pp. 191-238. MR 19, 50.

3. T. Kudo and S. Araki, Topology of $H_{n}$-spaces and $H$-squaring operations, Mem. Fac. Sci. Kyuashū Univ. Ser. A. 10 (1956), 85-120. MR 19, 442.

4. W. Browder, Homology operations and loop spaces, Illinois J. Math. 4 (1960), 347-357. MR 22 \#11395.

5. T. tom Dieck, Steenrod-Operationen in Kobordismen-Theorien, Math. Z. 107 (1968), 380-401. MR $39 \# 6302$.

6. E. Dyer and R. K. Lashof, Homology of iterated loop spaces, Amer. J. Math. 84 (1962), 35-88. MR 25 \#4523.

7. I. Madsen, Ph.D. Thesis, University of Chicago, Chicago, Ill. 1970.

8. J. P. May, The Dyer-Lashof algebra and infinite loop spaces (mimeographed notes).

9. - The homology of $F, F / O$ and $B F$ (mimeographed notes).

10. G. Nishida, Cohomology operations in iterated loop spaces, Proc. Japan Acad. 44 (1968), 104-109. MR 39 \#2156.

11. J. Stasheff, The image of $J$ as a space mod $p>2$ (mimeographed notes).

Yale University, New Haven, Connecticut 06520

University of Chicago, Chicago, Illinois 60637 